\title{
MEANDER-LIKE SHAPE OPTIMIZATION OF THE STATOR-ROTOR PLATFORM CAVITY
}

\author{
Paht Juangphanich ${ }^{1}$ \\ Purdue University \\ West Lafayette, IN, USA \\ pjuangph@purdue.edu
}

\author{
Guillermo Paniagua \\ Purdue University \\ West Lafayette, IN, USA \\ gpaniagua@purdue.edu
}

\begin{abstract}
Recent progress in additive manufacturing has enabled opportunities to explore novel stator rim geometries which can be implemented to improve cooling strategies in turbomachinery. This paper presents a simplified stationary geometry optimization strategy to produce enhanced stator-rotor cavity sealing and highlights main driving mechanisms.

The stator and rotor rims were designed using a design strategy based on inspiration from the meandering of rivers. A minimum thickness of $2 \mathrm{~mm}$ was maintained throughout the cavity to ensure a practical implementation. The computational domain comprised of the stator outlet, hub disk leakage cavity, and rotor platform was meshed using NUMECA Int. package, Hexpress. The numerical analysis required 3D Unsteady Reynolds Average Navier-Stokes to replicate vorticial structures using Ansys Fluent. The operating conditions were representative of engine-like conditions, exploring a wide range of massflow ratios from 1 to $3 \%$. The optimization yielded designs that provide $30 \%$ reduction in rear platform temperature while minimizing coolant massflow. The applicability of the design was compared against 3D sector in both stationary and in rotation.
\end{abstract}

\section{NOMENCLATURE}

$\begin{array}{ll}\mathrm{A} & =\text { Cell Area } \\ \mathrm{K} & =\text { Kelvin } \\ \dot{\mathrm{m}} & =\text { massflow rate } \\ \mathrm{P} & =\text { Pressure } \\ \mathrm{Q} & =\text { Q Criterion } \\ \mathrm{T} & =\text { Temperature } \\ \mathrm{V} & =\text { Velocity }\end{array}$

\section{Subscripts}

$0=$ Total flow quantity

$\begin{array}{ll}\text { ax } & =\text { Axial Direction } \\ \text { coolant } & =\text { Purge Flow } \\ \text { hot } & =\text { Mainstream } \\ \text { R } & =\text { Relative } \\ \text { RMS } & =\text { Root mean squared } \\ \text { STD } & =\text { Standard Deviation }\end{array}$

\section{Greek symbols}

$\begin{array}{ll}\alpha & =\text { purge exit angle } \\ \rho & =\text { Density }\end{array}$

\section{INTRODUCTION}

Advanced cooling strategies are needed to ensure the survivability of the turbine in harsh environments. Air is fed in between the stator rim and rotor platform to prevent hot gas ingestion. Studies have shown that the purge flow enhances the rotor hub vortex $[1,2,3,4]$. This phenomenon prevents coolant from reaching rear rotor platform near the pressure side. End wall cooling is often required to protect both the vane and rotor platforms, and in some instances, may be used to cool the blade region close to the platform, near the trailing edge. Friedrichs et al. [5] was one of the first researchers to document the significant pressure loss caused by the endwall cooling.

A study by Barigozzi et al. [6] investigated both the aerodynamic and thermal effects of endwall cooling on rotor cascade. They found that film cooling has negligible effects on aerodynamic performance when ejected along the pressure side endwall. However, the rear platform was not adequately protected due to cavity flow interacting with secondary flows. Adding more coolant requires more bleed flow from the compressor and is detrimental towards the overall engine efficiency; on the contrary, improving the purge design to provide endwall cooling may be a solution.

\footnotetext{
${ }^{1}$ NASA Glenn Research Center, Pathways Intern
} 
Redesigning the purge has its challenges such as preventing ingestion and controlling the interaction with the main flow. There has been a great deal of research on both the sealing effectiveness of different seal designs and on the interaction of purge flow with the main flow.

Double seal designs were been investigated by Zhou et al. [7]. Zhou and co-workers showed that double overlap seals with low aspect ratios (where seal gap is reduced) had the best sealing effectiveness. Additionally, there have been studies on how to parameterize and optimize purge designs. Moon et al. [8] parameterized a rectangular cavity on the stator side of a double overlap seal configuration and studied the sealing effectiveness and the influence the seals had on minimizing passage vortex. Popović and Hodson [9] investigated the effect of changing the overlap of the rim seal geometry on sealing effectiveness and the effect on the passage vortex using a linear experimental rig. In 2013, Popović and Hodson [10] experimentally tested a variety of seal designs that are similar to what is currently used in industry. They discovered that an inclined ejection does not result in a strong leakage vortex. Injecting coolant flow at a shallower angle provides better coolant to the endwall.

The study of rim seal design configurations has primarily been focused on preventing ingestion. However, the effect of purge flow on the rotor should also be considered. McLean et al. [11] studied the effect of purge flow on the performance of the turbine. They showed that a $1 \%$ change in cooling can significantly affect the total-total efficiency of the turbine. Pau et al. [2] measured the effect of purge flow on the Nusselt number of the rotor platform. They determined that rotor platform cooling has minimal effects on the suction side and trailing edge of the rotor. Barigozzi et al. [6] also suggested that this is due to the coolant flow from the hub disk being captured by the rotor hub passage vortex which moves the coolant away from the rotor endwalls. Suryanarayanan et al. $[12,13]$ showed using pressuresensitive paint that coolant supplied by purge flow did not provide sufficient coolant to the pressure side of the rotor.

While many researchers investigate the rim seal's performance in 3D experiments and simulations, other researchers have studied the rim seal in 2D and related their findings to 3D. Cao et al. [14] modeled the cavity in 2D and 3D in different sectors sizes. They found that the trend of their 2D simulation with an axial gap of $2 \mathrm{~mm}$ agreed with experimental results. Mirzamoghadam and Xiao [15] were able to match the 2D CFD with experimental test data for temperature and pressure within the seal. However, they could not delineate the effects of rotation.

This paper aims to simplify the design and analysis of rim seals through a quasi 2D optimization. The paper explores smooth and indented geometries to quantify potential aerothermal benefits. However, this paper does not address the actual manufacturability of the geometries, nor the mechanical and thermal stresses induced in the rotating components. This paper uses optimization tools to explore alternate purge designs that could provide cooling further downstream than conventional designs. One of the critical aspect of any optimization strategy is the parameterization of the geometry. In this paper we explore a new design approach based on shapes that are inspired by the meandering of rivers.

\section{PARAMETERIZATION OF THE CAVITY}

\subsection{Meander Line}

The purge geometry is constructed using 3 components: the meander line, the stator rim, and the rotor rim (Fig. 1). The meander line is designed using 4 Bezier curves. Letters " $A$ " and "B" denote the thickness of the meander. The first Bezier begins at point 1 and ends at point 4 . Points 2 and 3 are used to control the starting and ending first derivatives of the Bezier curve. Intermediate points indicated by white filled circles in between points 3 and 4 are used to straighten out the Bezier curve and create spacing between the curves.

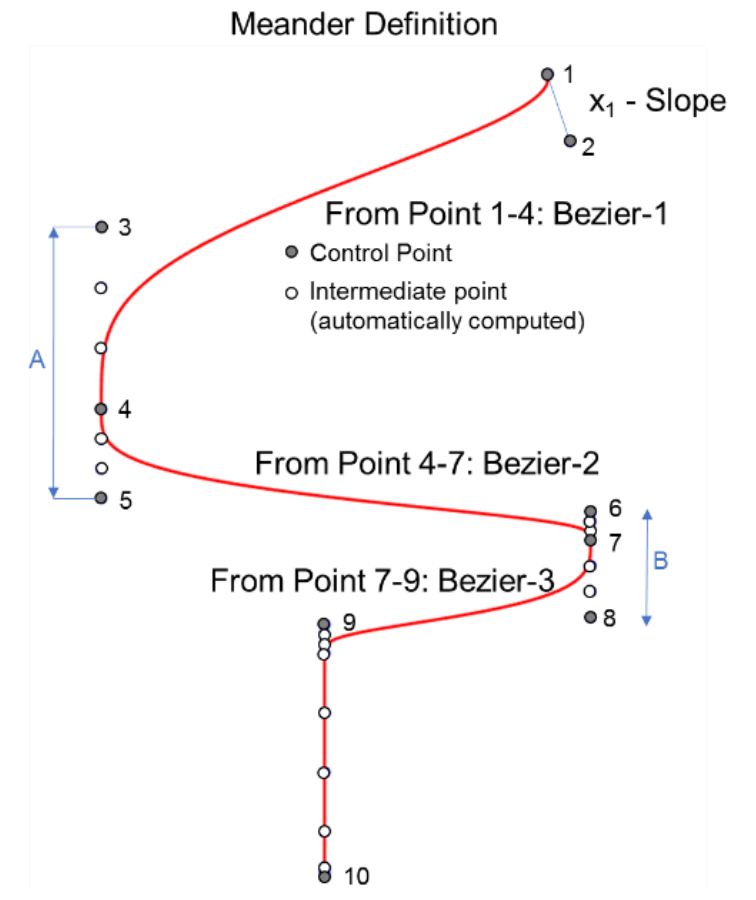

Fig. 1. Meander Line. Flow moves from bottom (Point 10) to top (Point 1).

Four parameters are needed to construct the first Bezier curve (Bezier-1): the slope which determines where point 2 lies, the $\mathrm{x}$ and $\mathrm{y}$ location of point 4 , and point " $\mathrm{A}$ " which is the thickness of the meander. Bezier 2 is constructed using 3 parameters: the thickness of the meander in Bezier 1, "A", combined with the $\mathrm{x}$ and $\mathrm{y}$ location of point 7 . A separate thickness " $\mathrm{B}$ " is used to control the $2^{\text {nd }}$ meander thickness. The $3^{\text {rd }}$ Bezier is automatically defined from point 7 to point 9 . Point 8 is created using the thickness "B." Points 9 and 10 are at a fixed location. In total, 7 parameters are used to design the meander line. 


\subsection{Stator and Rotor Rims}

Stator and rotor rims are formed by imposing thickness distributions along the meander line (Fig. 1) from exit (Point 1) to inlet (Point 10). Stator and rotor rim thickness distributions are created using splines with 6 control points used to describe the thicknesses along the stator and rotor rims (Fig. 2-Left). The $\mathrm{y}$ coordinate represents the thickness, the $\mathrm{x}$ coordinate is the $\%$ along the purge from the purge exit to the purge inlet. The last 2 points for both stator and rotor rim are fixed to maintain same purge exit thickness. The first 4 control points are free to move in the y direction adding thickness to the design (Fig. 2-Right); however, their $\mathrm{x}$ direction components are fixed to limit the parameter space.

The rims are created using the thickness distribution (Fig. 2Left), and points are evenly spaced out along the meander line. The points are then shifted perpendicular to the meander line by a distance defined using the stator and rotor rim thickness (Fig. 2- Left). Fig. 2-Right shows the applied rotor and stator rims; the dip in the thickness at point 2 in Fig. 2-Left creates a contraction in the purge whereas points with higher $y$-values expand the purge. A total of 8 control points are used to create the rim thickness.

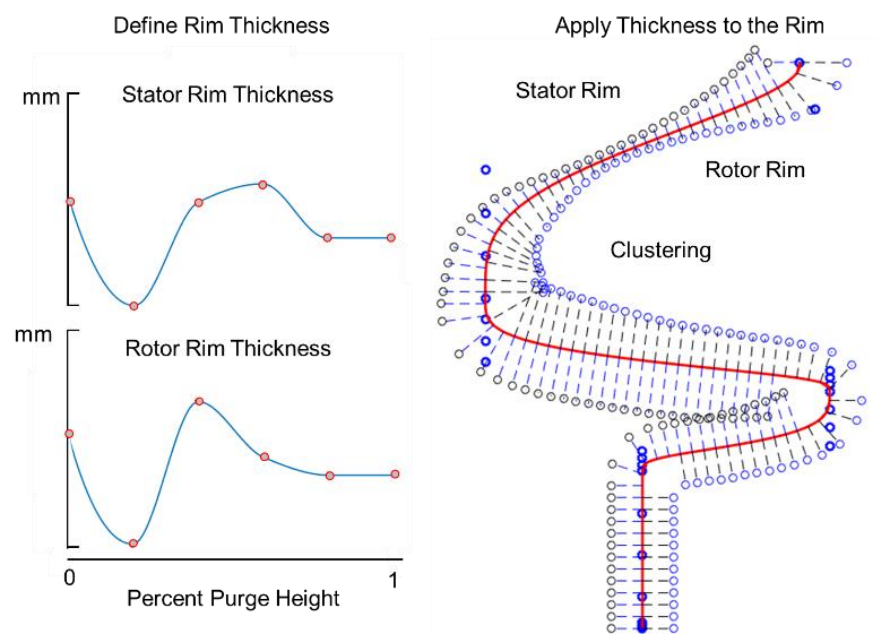

Fig. 2. Left Stator and Rotor Rim Thicknesses. Right. Purge after thicknesses are applied. After thicknesses are applied, there can be intersection and parts where minimum purge separation are not maintained.

The lowest limit of axial spacing was set to $2 \mathrm{~mm}$. The limit is based on a baseline configuration inspired from a research program performed in a rotating facility at the von Karman Institute [2] and from Roy et al. [16]. The implementation of a thickness distribution may lead to axial stator-rotor gaps greater than the lowest axial spacing limit $(2 \mathrm{~mm})$. In addition, there are parts along the purge where points intersect. To solve these problems, additional thicknesses are added to the points that violate the spacing constraints. Then a check is performed to remove troublesome points that intersect each other. The final purge design is shown in Fig. 3-Left. The design is then connected to the platform (Fig. 3-Right). A Bezier curve is used to blend smooth the connection and remove any sharp corners (Fig. 4).
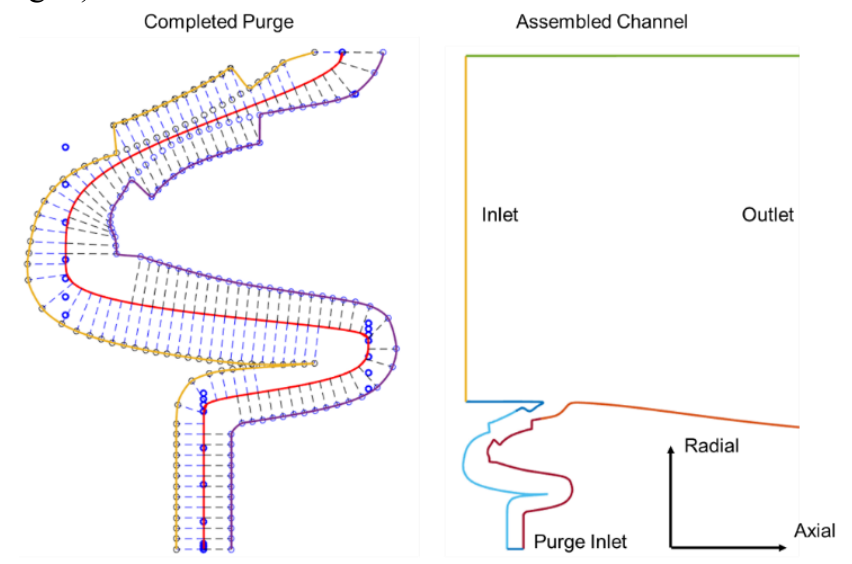

Fig. 3. Left: Final Purge design. Right: Purge connected to the channel

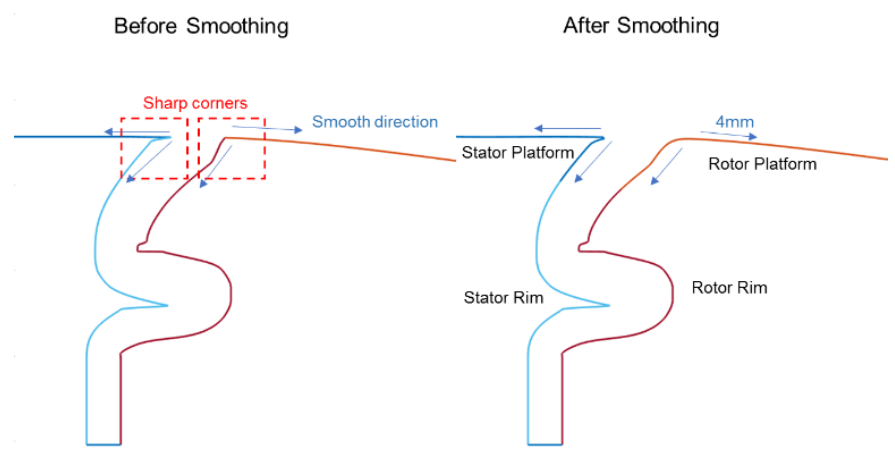

Fig. 4. Smoothing of sharp corners. A Bezier curve is constructed using points $4 \mathrm{~mm}$ along the rotor platform and rotor rim of the pure to provide smoothing of the purge exit.

\section{OPTIMIZATION APPROACH}

\subsection{Strategy}

The true physics of the stator-rotor cavity in $3 \mathrm{D}$ with rotation is an aerothermal challenge that requires computationally onerous simulations. Instead, in an attempt to simply the problem, we first examined a slice of the flow, by considering a pure 2D case without rotation. This enabled the optimization of the geometry in 2D. Then a few selected/optimized geometries were investigated in $3 \mathrm{D}$, in the stationary frame as well as in the rotating frame. This approach allowed us to reduce the computational time. Additionally, this strategy enabled a more detailed understanding of the physics, revealing where the flow is predominantly $3 \mathrm{D}$, or $2 \mathrm{D}$, and the effects of rotation. Furthermore, the comparisons with detailed correlations of pipe flow revealed separated regions within the purge geometry.

Our 2D design strategy was to apply the parameterization (Fig. 5-Top) strategy to design a purge geometry that minimizes the massflow rate while cooling the rear part of the rotor platform. The overall methodology is shown in Fig. 5-Bottom. It consists of coupling a multi-objective differential evolution 
optimizer to a geometry creation routine. The geometry is then exported to an unstructured mesher and solved using fluent. The results are then post processed and sent to the optimizer.

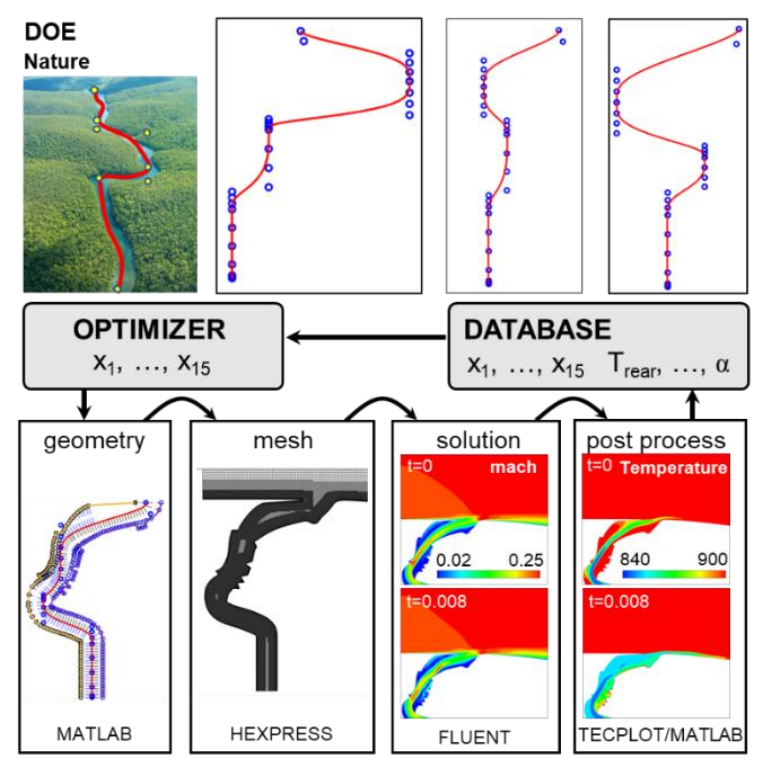

Fig. 5 Overall Strategy

The optimizer used is CADO [17]. CADO uses a database of individuals twice the size of the population in its restart population. Individuals stored in this database are paired using crossover and mutation to generate the new population of designs. The population is then evaluated and ranked using the NSGA II algorithm to rank the individuals [18]. Fitter individuals are stored for the creation of the new population whereas others are discarded. The multi-objective optimization routine uses 30 individuals per population. The population size was chosen as a balance between speed, evaluation time, and geometric variability. The optimization routine manages the creation of the geometry all the way to the post processing.

Geometry creation is performed using MATLAB. A total of 15 design parameters are used to create the geometry -7 for the meander line and 8 for the rim thicknesses. The meander line is first created then stator and rotor rims are applied. The geometry is then exported to a file that is read by HEXPRESS. HEXPRESS creates an unstructured 3D hexahedral mesh containing 2kplanes in the tangential direction with symmetry boundary condition. The grid (Fig. 6) is exported to the solver, Ansys Fluent 18.1. Fluent evaluates the computational domain in steady state for 5000 iterations before transitioning to unsteady evaluation for 800 timesteps. The transition to unsteady CFD is required to be able to compute the time averaged quantities required in the optimization step. In the last step, the results are post processed using TECPLOT and the results are sent back to the optimizer.

\subsection{Computational Domain and Boundary Conditions}

The domain is shown in Fig. 6. The geometry is from Juangphanich et al. [19]. The stator and rotor geometries are not used in the analysis. The domain has symmetry boundary conditions on the side walls, and there is no rotation applied to the rotor platform. Wall spacing of $1 \mathrm{E}-7 \mathrm{~m}$ is used to create a viscous layer on the stator and rotor platform and inside the purge.

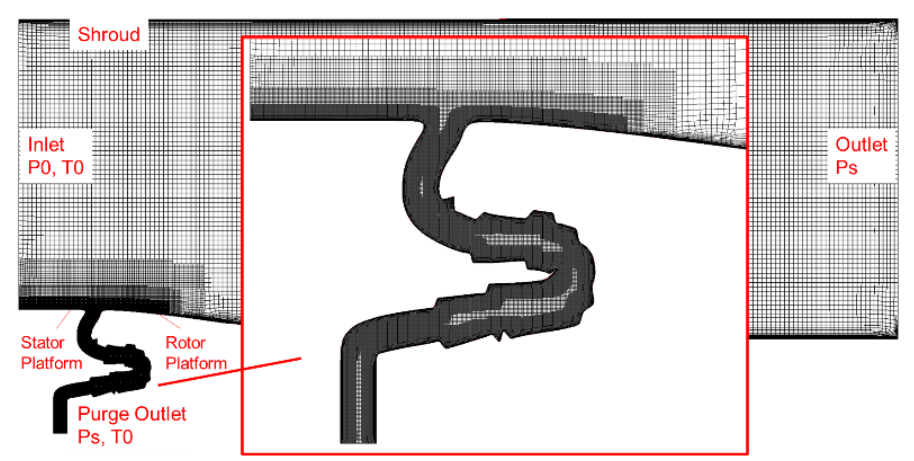

Fig. 6. Computational Domain mesh generated using HEXPRESS

The computational domain was solved at engine level relative rotor inlet conditions. Inlet relative total pressure $\left(\mathrm{P}_{01 \mathrm{R}}\right)$ of 30 bars and an Inlet relative total temperature $\left(\mathrm{T}_{01 \mathrm{R}}\right)$ of $1700 \mathrm{~K}$ was used. The exit static pressure $\mathrm{P}_{\mathrm{S} 2}$ of 28.9 bar was assumed such that the relative mach number at the purge inlet was 0.3 and there was a massflow ratio of $1.4 \%$ in the baseline simulation. Purge outlet total temperature was assumed to be $850 \mathrm{~K}$ maintaining a $T_{\text {hot }}$ to $T_{\text {coolant }}$ ratio of 2 . Stator and rotor platform were isothermal with a wall temperature of $1133 \mathrm{~K}-\mathrm{T}_{\text {hot }}$ to $\mathrm{T}_{\text {wall }}$ of 1.5. The $y+$ was less than 0.3 inside the cavity and less than 1 along the rotor platform.

The solver evaluated the domain using Reynolds Average Navier-Stokes (RANS) for 5000 iterations before transitioning to Unsteady Reynolds Average Navier-Stokes (URANS) for 800 timesteps at 1E-5 seconds per timestep. The k-omega SST turbulence model and ideal gas assumptions were used. Inlet turbulence level of $15 \%$ was assumed.

\subsection{Assessment of the Convergence and Grid}

Grid sensitivity was evaluated by solving the URANS simulation at 4 grid levels. The last 100 timesteps were massflow weighted averaged to determine the temperature above the rear and front of the rotor platform using equations (1) and (2). The locations above the platform are indicated in Fig. 7. Fig. 7 shows the massflow-averaged rear temperature above the rear platform (Fig. 7: Rectangle). Both fine and finer meshes were plotted against the number of nodes. There was a large difference between the coarse and medium meshes; however, the fine and finer meshes show a difference of $0.8 \mathrm{~K}$. The finer grid was selected based on the evaluation time considering computational resources available. Fig. 8 shows the fluctuation in temperature 
plotted against time for the weighted average rear temperature. The temperature is periodic after $6 \mathrm{~ms}$ as indicated by the zoomed-in plot.

$$
\begin{gathered}
T_{\text {rear }}(t)=\frac{\sum_{i=1}^{N_{\text {cells }}}\left(A_{\text {cell }} \rho V_{a x} T\right)_{i}}{\sum_{i=1}^{N_{\text {cells }}}\left(A_{\text {cell }} \rho V_{a x}\right)_{i}} \\
T_{\text {rear }}=\sum_{t=\left(T_{\text {end }}-100\right)}^{T_{\text {end }}} T_{\text {rear }}(t)
\end{gathered}
$$

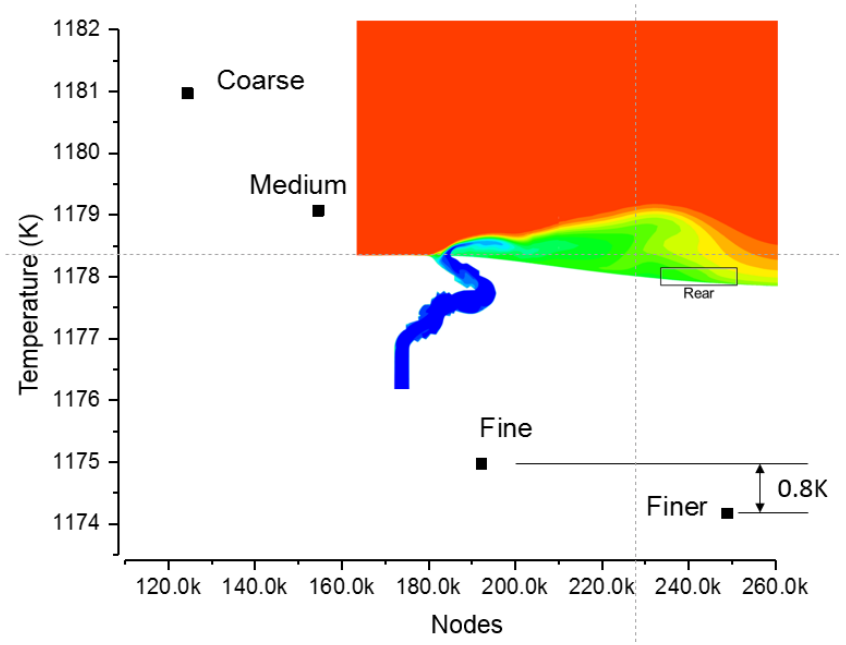

Fig. 7. Mesh Sensitivity

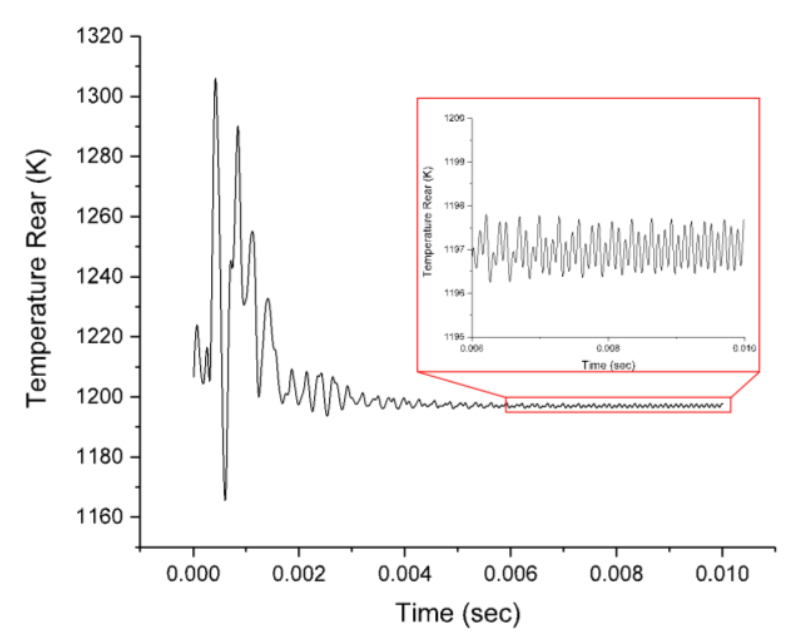

Fig. 8. Unsteady Rear Temperature. Rear temperature is periodic after $6 \mathrm{~ms}$

The selected grid was then assessed for unsteady convergence. The periodicity of the rear temperature flowfield was verified using the method of Clark and Grover [20]. This method evaluates the phase, amplitude, mean value, and the cross correlation for each cycle. The solution is considered periodic when a negligible difference between two consecutive cycles has been identified. The minimum level convergence reached in the mesh sensitivity in our study was 0.9561 .

\section{RESULTS}

\subsection{Effect of Geometry on Platform Temperature}

Fig. 9 presents the results of the optimization. Ten populations were analyzed with 30 individuals per population. Each point in the figure is an individual and represents a unique design. Rear temperature was averaged within the region above the rotor platform (Fig. 7-Box) using a weighting of cell area and the massflow and plotted against mass fraction (Eqn. 3) - purge massflow divided by the inlet massflow. Each point is contoured using the blowing ratio (Eqn. 4). Blowing ratio increases with massflow rate. The baseline, which is a double overlap seal, is indicated with a diamond near the top of the graph; the straight purge is represented by a diamond near the bottom. The red line represents the pareto front. The pareto illustrates the tradeoff between the designs with lowest rear platform temperature and minimum purge massflow ratios.

$$
\text { Massflow Ratio }=\frac{\dot{m}_{\text {Purge Inlet }}}{\dot{m}_{\text {Inlet }}}
$$

Purge designs that feature low massflow ratios are characterized by 2 meanders. Design A offers better cooling than the baseline for a small penalty in purge massflow. It does this by ejecting the purge flow against the flow direction. Design $\mathrm{B}$ provides a $150 \mathrm{~K}$ difference over the baseline for a $0.5 \%$ increase in massflow. In this design, flow is ejected in the direction of the mainstream flow. This reduces the mixing and provides better cooling to the platform. Designs $\mathrm{C}$ uses $2 \%$ more massflow than the baseline and provides $200 \mathrm{~K}$ lower temperature on the rear platform.

$$
\text { BlowingRatio }=\frac{\rho_{\text {coolant }} V_{\text {coolant }}}{\rho_{\infty} V_{\infty}}
$$




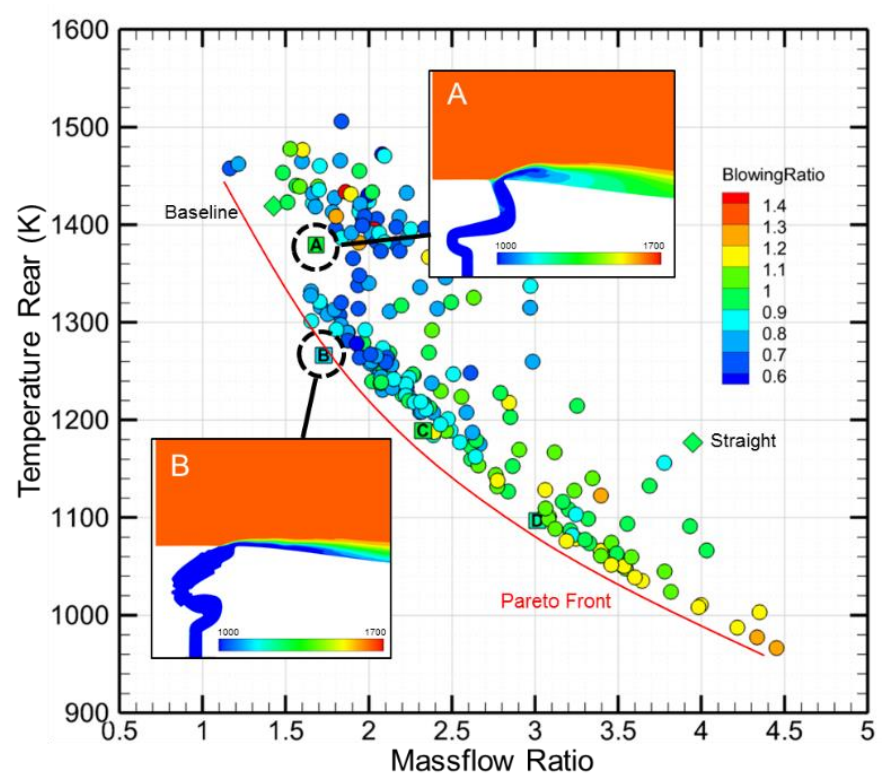

Fig. 9. Rear Temperature vs. Massflow Ratio contoured by Blowing Ratio

Fig. 10 is the same plot as Fig. 9 but contoured with temperature in the front of the platform averaged over the last 100 timesteps. Designs that provide the best cooling to the rear are also the best at cooling the front. Design B provides a $130 \mathrm{~K}$ drop in front platform temperature over the baseline design. Design C and D provide $200 \mathrm{~K}$ and $280 \mathrm{~K}$ improvement over the baseline, respectively.

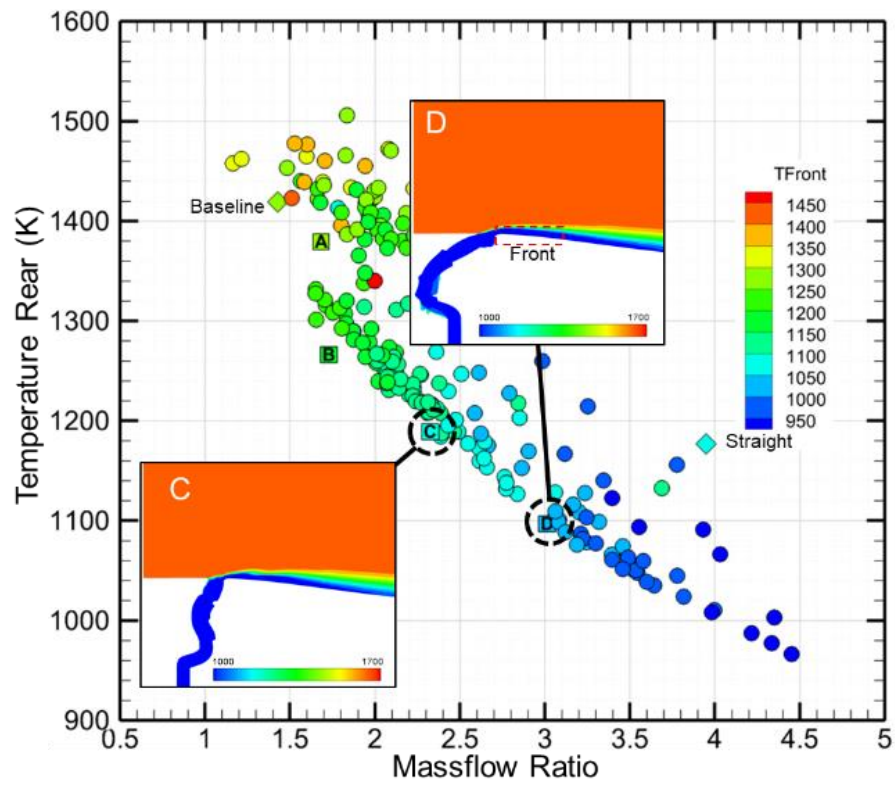

Fig. 10. Rear Temperature vs. Massflow Ratio contoured by Front Temperature

Fig. 11 shows a contour of the turbulence kinetic energy (TKE) plotted with lines of constant Q Criterion for the selected designs at the top and at the bottom of the pareto front, $\mathrm{A}$ and $\mathrm{D}$, at two time instances. Vorticity is identified by lines of constant $\mathrm{Q}$ calculated using TECPLOT. Q represents the balance between shear strain rate and vorticity magnitude [21]. Vortices are identified in areas where vorticity magnitude is greater than the magnitude of the rate of strain $[22,23]$. Fig. 12 shows the presence of the vortex plotted with contour of temperature at the same time instances as Fig. 11.

Areas with high TKE indicate the presence of strong mixing between main flow and purge flow which increases the temperature along the rotor platform. Design A ejects the purge flow against the main flow. This creates a large vortex that mixes with the purge flow and detaches as it travels downstream (Fig. 11-Top). The effect of mixing increases the temperature along the rotor platform (Fig. 12-Top).

Unlike Design A, Design D purges more massflow, but due to its ejection angle there is less mixing with the main flow. The vortex at the purge exit is shed and decays as it travels downstream. As a result, the purge flow is able to travel further down the platform than in Design A.

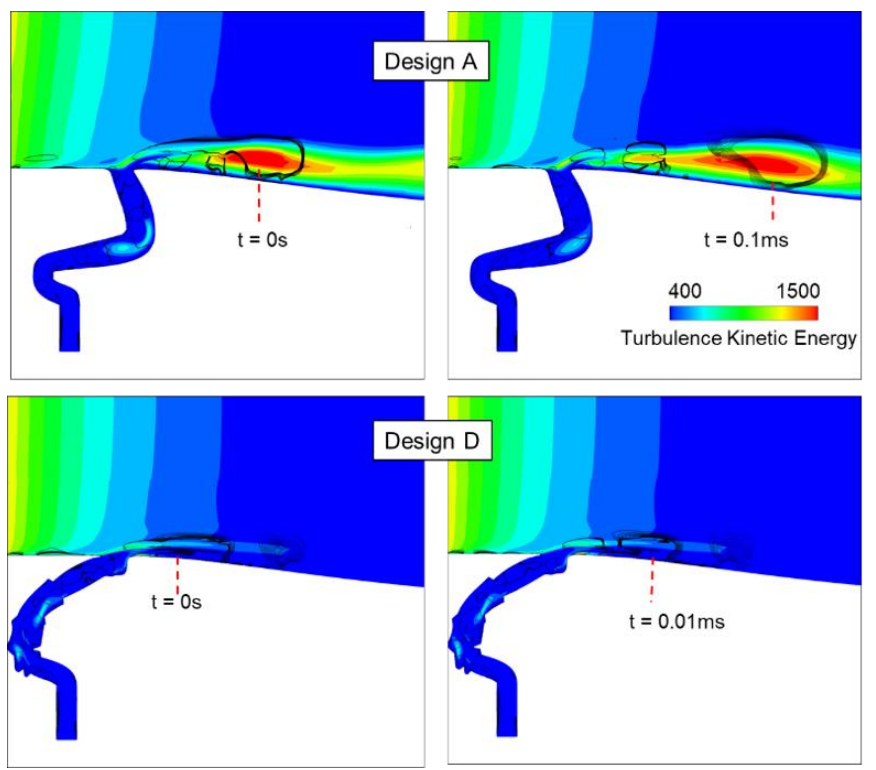

Fig. 11.Turbulence Kinetic Energy. Mixing is indicated by high TKE; designs that provide better cooling shows less mixing. 


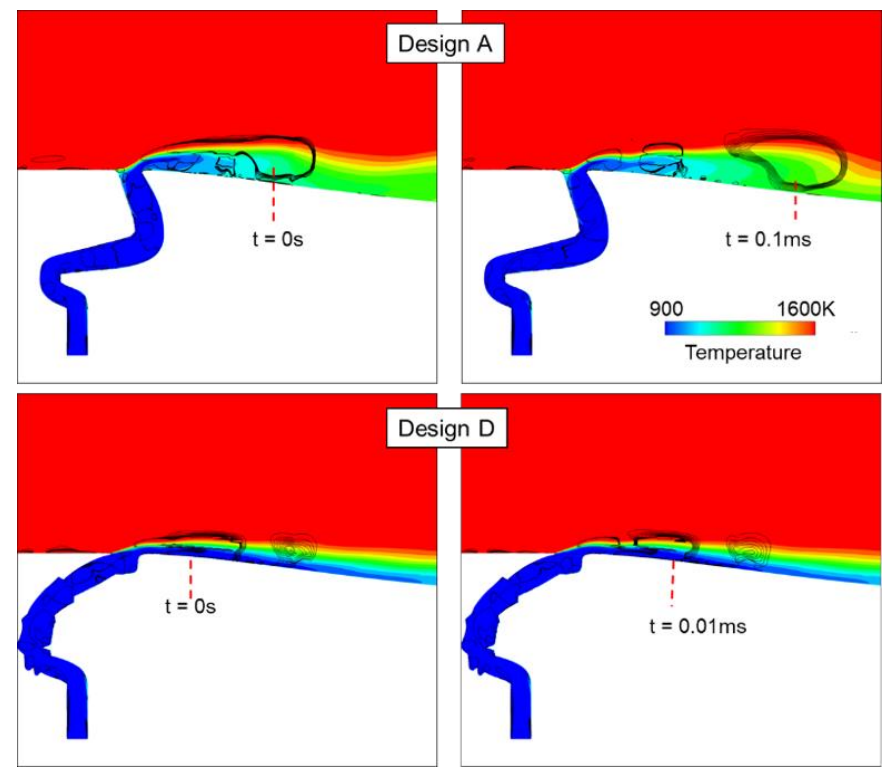

Fig. 12. Temperature plotted with constant lines of $Q$ Criterion. The presence of vorticial structures is presented in circular lines.

\subsection{Purge Temperature Stability}

Stability of temperature of the flowfield in the rear part of the platform was measured using the deviation of the root mean squared (Eqn. 5). The RMS and standard deviation of RMS were evaluated using the temperature of the flow above the rear platform for the last 100 timesteps. Fig. 13 displays the plot of the stability of each individual purge design vs. massflow rate contoured with the standard deviation of temperature.

A trend between the massflow rate and temperature stability can be seen. Designs that allow more massflow result in less fluctuations in rear platform temperatures. Designs that limit the massflow rate have higher temperature fluctuations. Finally, designs that eject flow against the mainstream show higher temperature fluctuations. The red line shows a predicted trend of stable designs vs. massflow rate.

$$
T_{S T D}=\sqrt{\frac{1}{N} \sum_{i=1}^{N}\left(T_{R M S}-T_{i}\right)^{2}}
$$

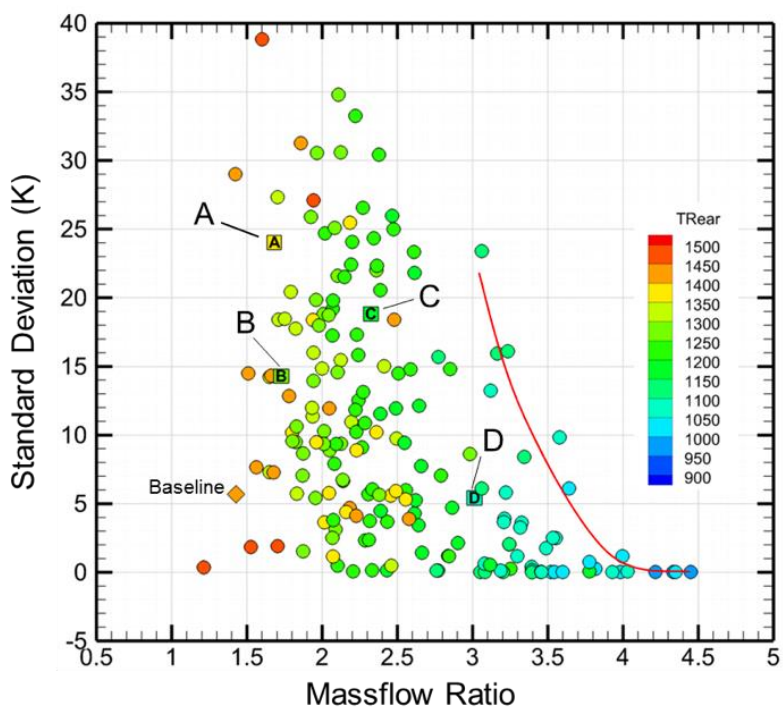

Fig. 13. Standard Deviation of temperature vs. Massflow contoured with standard deviation of rear platform temperature

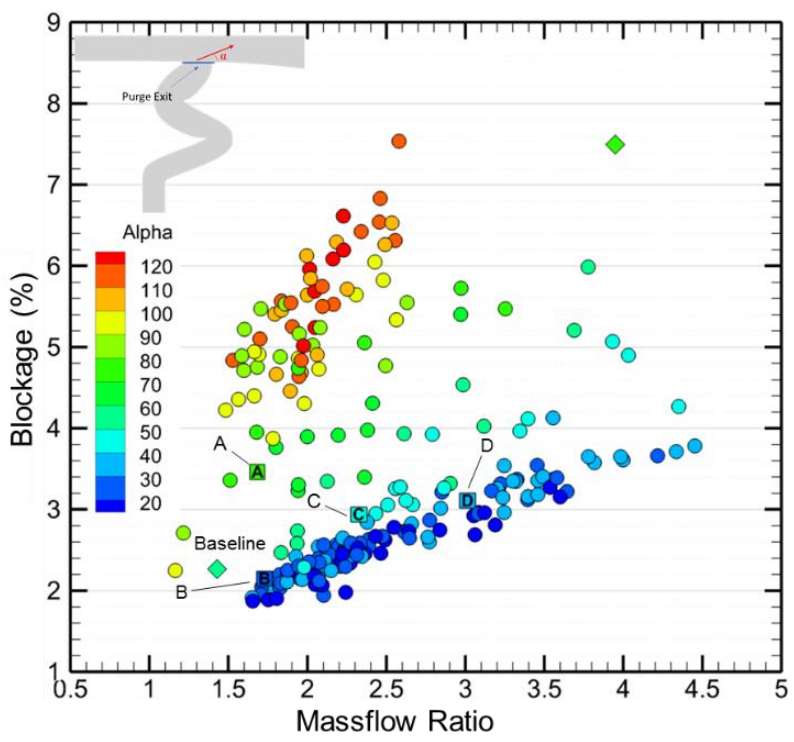

Fig. 14. Blockage vs. Massflow contoured with Purge Exit Angle. Low blockage designs feature exit flow angles of 20-30.

\subsection{Effect of Blockage and Flow Exit Angle}

The effect of blockage was compared for all purge designs. Blockage (Eqn. 6) was evaluated by the massflow rate at the inlet of each design divided by the massflow rate of the channel if there was no purge. Flow exit angle alpha was determined by taking a plane at the purge exit and computing the angle using the exit velocities (Eqn. 7).

Fig. 14 shows a plot of the blockage plotted against the massflow rate and contoured with flow exit angle. Blockage is reduced for designs that exit at 20-30 degrees; however, as massflow increases to $4-5 \%$, the blockage increases and the exit angle also rises to around 30 degrees. 


$$
\begin{aligned}
\text { Blockage } & =1-\frac{\dot{m}_{\text {inlet }}}{\dot{m}_{\text {no purge }}} \\
\alpha & =\tan ^{-1} \frac{V_{y}}{V_{x}}
\end{aligned}
$$

The blockage was also compared with the stability of the purge exit angle in Fig. 15. Stability was assessed using the standard deviation. Steadier designs are those that eject at shallower angles of 20-30 degrees. Designs that eject at higher angles are more unstable also cause higher blockage of the main flow.

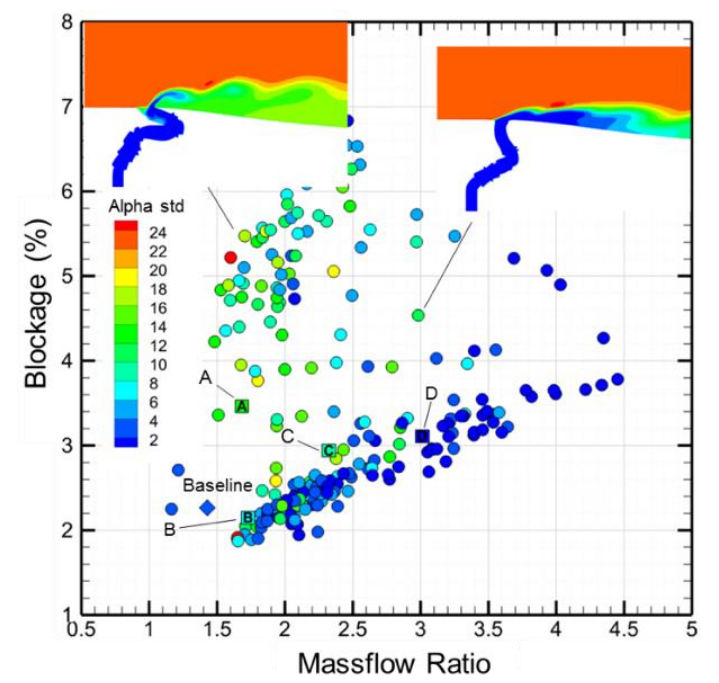

Fig. 15. Blockage vs. Massflow contoured by standard deviation of alpha

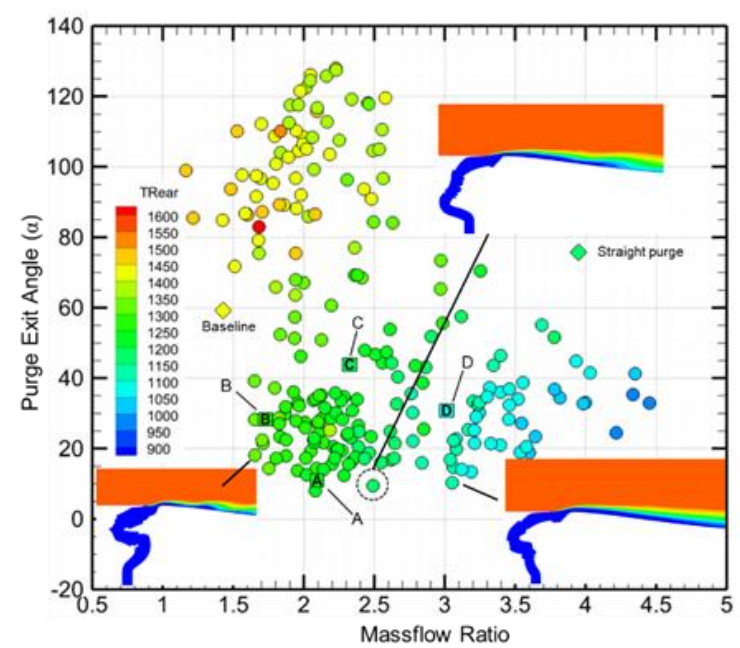

Fig. 16. Purge Exit Angle vs. Massflow contoured with rear platform temperature

Fig. 16 shows how well the purge flow exit angle cools the rear platform temperature as massflow increases. Designs with low massflow rates and flow ejection angles at around 20 degrees tend to cool the rear platform to temperatures to around 1150$1350 \mathrm{~K}$. However, if the angle is kept at a constant 20 degrees but the massflow increases to $2.5-3.5 \%$, this results in designs that improve the cooling to around $1100 \mathrm{~K}$. Increasing the massflow more than $3.5 \%$ can reduce the rear temperature further at the cost of increasing blockage, as indicated by tracking Design D in both Fig. 16 and Fig. 14.

\subsection{Nusselt Number comparison with Pipe Flow}

For a few selected geometries, the same geometry was investigated at two different wall temperatures; the entire cavity was set isothermal at $1133 \mathrm{~K}$, and then $1300 \mathrm{~K}$. The adiabatic convective heat transfer was then computed following the approach described by Pinilla et al. [24]. Assuming a linear trend of the heat flux as a function of the wall temperature, the Nusselt number was computed using equations (8) and (9).

$$
\begin{aligned}
& h=\frac{Q_{2}-Q_{1}}{T_{1}-T_{2}} \\
& N u_{D}=\frac{h D_{i}}{k_{\text {film }}}
\end{aligned}
$$

This geometric-specific Nusselt is benchmarked with wellknown correlations of local Nusselt number for developing flow in ducts subject to uniform heat flux, following the approach presented by Solano et al. [25]. The correlation from Churchill and Ozoe [26], Eqn. (10), was used to estimate the laminar $\mathrm{Nu}_{\mathrm{D}}$ with $n$ set to 10 . Graetz number $(\mathrm{Gz})$ was computed using the hydraulic diameter of each station in Fig. 17 and 18 divided by the station's location along the curve.

$$
\begin{aligned}
& \frac{N u_{D}+1}{5.364\left[1+(G z / 55)^{\frac{10}{9}}\right]^{\frac{3}{10}}} \\
& =\left(1+\left(\frac{G z / 28}{\left[1+(\operatorname{Pr} / 0.0207)^{\frac{2}{3}}\right]^{\frac{1}{2}}\left[1+(G z / 55)^{\frac{10}{9}}\right]^{\frac{3}{5}}}\right)^{\frac{n}{2}}\right) \\
& G z=\frac{\operatorname{Re}_{D} \operatorname{Pr} D_{H}}{x_{i}}
\end{aligned}
$$

Correlation from Bhatti and Shah [27] was used to estimate turbulent $\mathrm{Nu}_{\mathrm{D}}$ where $\mathrm{Nu}_{\infty}$ denotes the fully developed Nusselt number.

$$
\frac{N u_{x}}{N u_{\infty}}=1+0.234\left(\frac{D_{H}}{x_{i}}\right)^{0.76}
$$

Fig. 17 shows the Nusselt number along the rotor rim of the straight purge as a function of the radial position, measured from the purge inlet. The numerical results are compared with the laminar and turbulent pipe flow correlations. For this baseline configuration with straight purge, the Nusselt number exhibit a performance close to the flow in a laminar pipe.

Fig. 18 displays the Nusselt number distribution for the configuration $\mathrm{B}$; the abscissa is the curvilinear coordinate measure following the radial direction as sketched in Fig. 18right. The inlet part of the cavity exhibits a trend similar to the laminar pipe. However, at locations 4 and 6 , where we expect 
the reattachment of the recirculation bubble, the Nusselt number approaches the turbulent level.

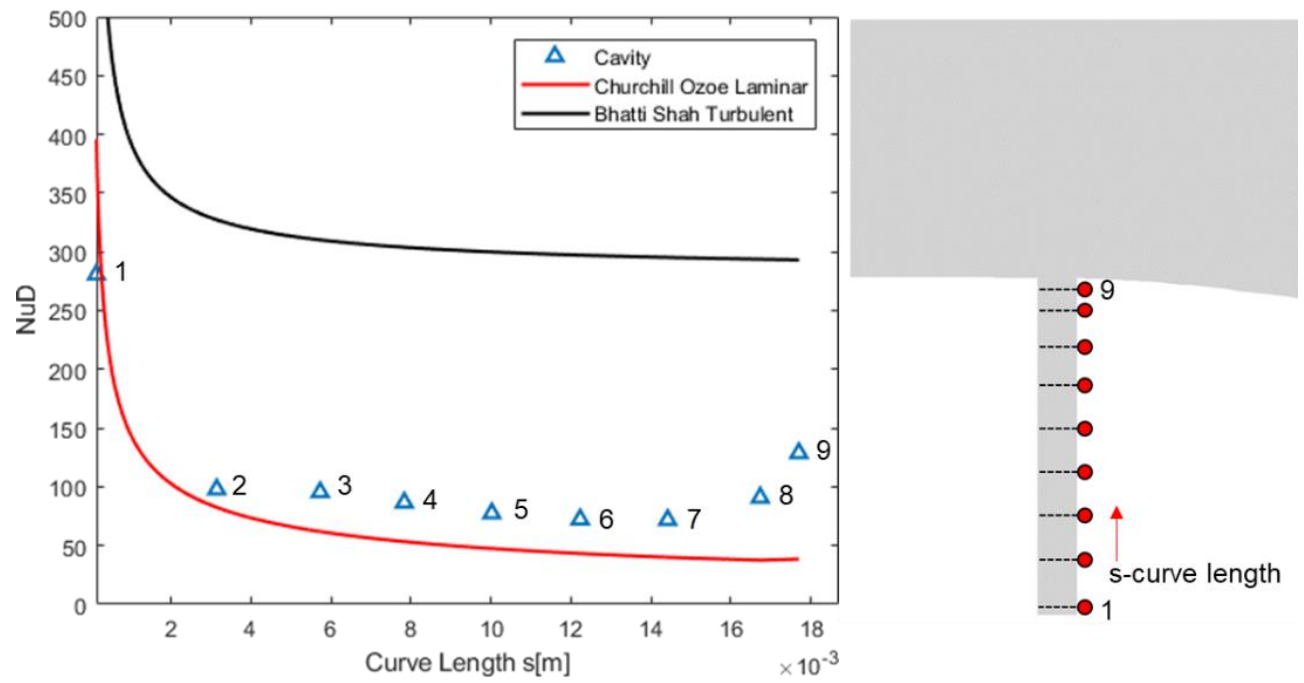

Fig. 17. Baseline Cavity and Pipe flow comparison. Left: Nusselt distribution along cavity walls. Right: stations where data was extracted
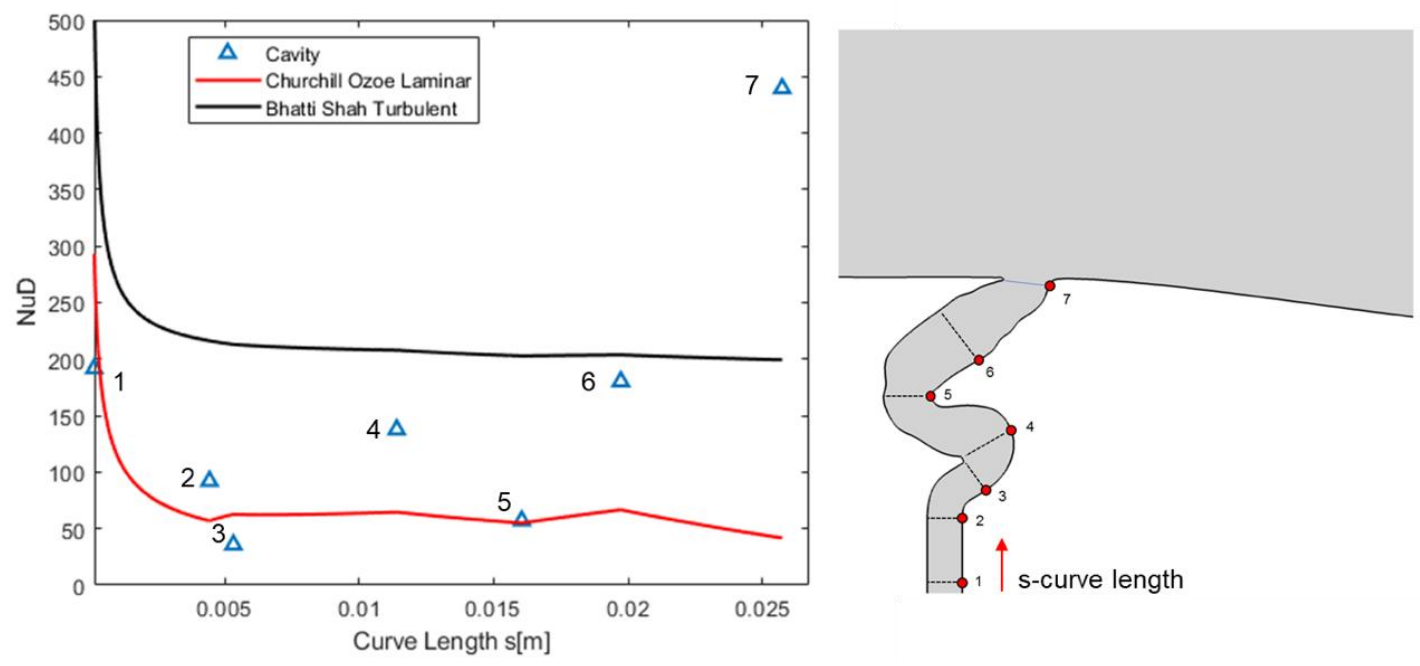

Fig. 18. Design B Cavity and Pipe flow comparison. Left: graph of Nusselt distribution along the cavity wall. Right: stations where data was extracted

\subsection{Smoothness Comparison}

The geometries investigated may not necessarily be smooth. Few geometries in the optimization process are smoothed, e.g. Design A. To make these geometries more suited for manufacturing, a smoothing function can be applied to the surface. Eqn. 13 is an example of the smoothing function used to generate the smooth geometry in Fig. 19 on the bottom right. The function iterates over all the points on the surface and averages the point $p$ with its neighbors. The moving average function ran twice to generate the smooth geometry.

$$
p_{i}=\frac{p_{i-1}+p_{i}+p_{i+1}}{3}
$$

In the investigated cases, the smoothed geometry resulted in a higher outlet velocity, and therefore higher Reynolds numbers. Thus, smoothness increases the heat transfer between cool purge flow and the hot walls within the cavity. The effect becomes more pronounced towards exit. This impacts the cooling of the platform. Fig. 20 shows the heat flux along the platform. Positive heat flux indicates fluid cooling the wall, while negative heat flux indicates fluid heating up the wall. The smooth geometry was able to eject cool flow further along the platform than the rough design. 

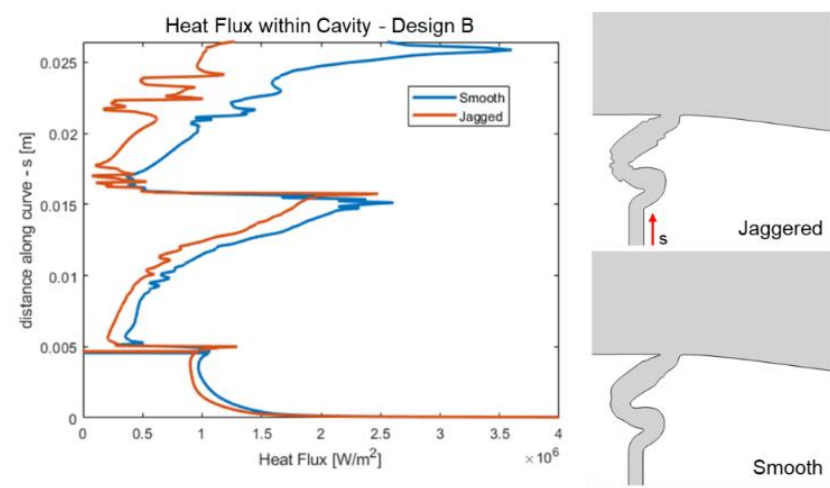

Fig. 19. Left: Heat Flux within the Cavity comparison with smooth geometry. Right Top: Jagged. Right Bottom: Smooth

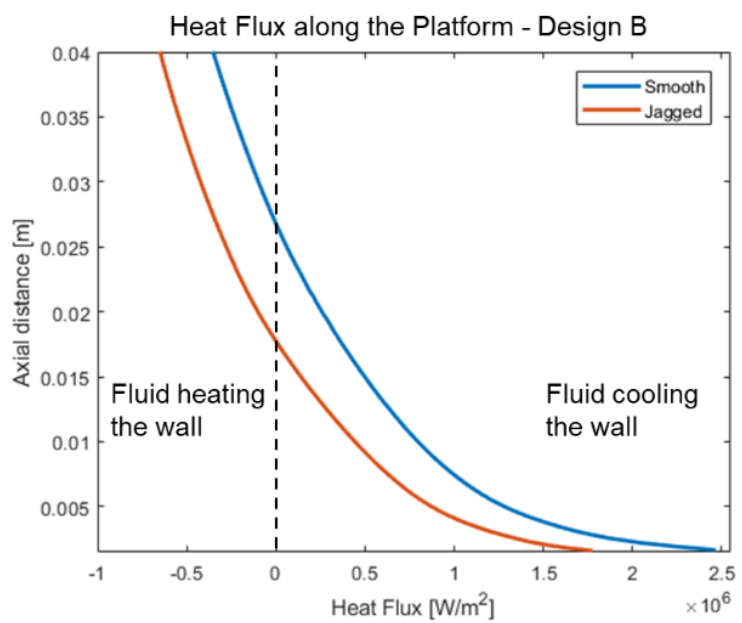

Fig. 20. Heat Flux along the Platform. Smoothed vs. Rough comparison

\subsection{D/3D Stationary and Rotation Comparison}

The 3D computational domain shown in Fig. 21 was created using Autodesk Inventor by revolving the 2D design about the axial direction, along a sector. The total size of the mesh is above $9 \mathrm{M}$ cells, with the same inflation layer as the $2 \mathrm{D}$ mesh. Periodicity was applied at the two sides of this 3D domain. The same cell sizes used in the 2D simulation were used to create this 3D geometry with ANSYS. The element type was changed to tetrahedrons to match periodicity along the tangential walls. The simulations were performed with the Reynolds Averaged Navier Stokes solver. The rotational simulation was executed in the rotor's reference frame by applying a rotational speed of 7000 RPM to the stator side of the purge.

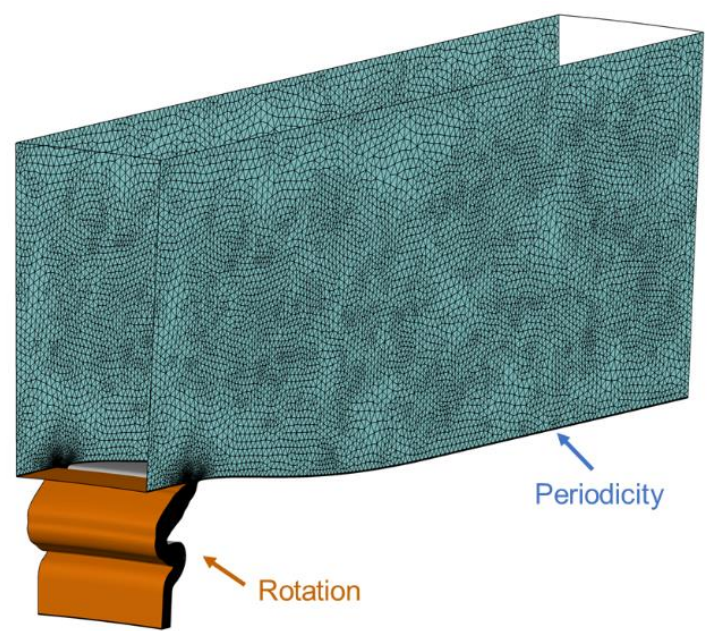

Fig. 21. Design B. Rotation is applied to the stator side of the purge

Fig. 22-Left displays the heat flux along the radial location within the cavity, the $2 \mathrm{D}$ results are compared against the $3 \mathrm{D}$ stationary and $3 \mathrm{D}$ rotational. The three-dimensional data was obtained by performing a pitch-wise average at each radius. The 3D stationary results (red triangles) compare well with the $2 \mathrm{D}$ case. When rotation is added, the heat flux may deviate from the $2 \mathrm{D}$ simplified assumption by about $16 \%$ of the mean level; however, the trend remains the same for both the $2 \mathrm{D}$ and the 3D rotational. Fig. 22-Right depicts the heat flux on the rotor platform, along the axial direction. The distribution of the $2 \mathrm{D}$ simulation agrees well with the $3 \mathrm{D}$ results; the effect of rotation in the platform is less important than within the cavity.
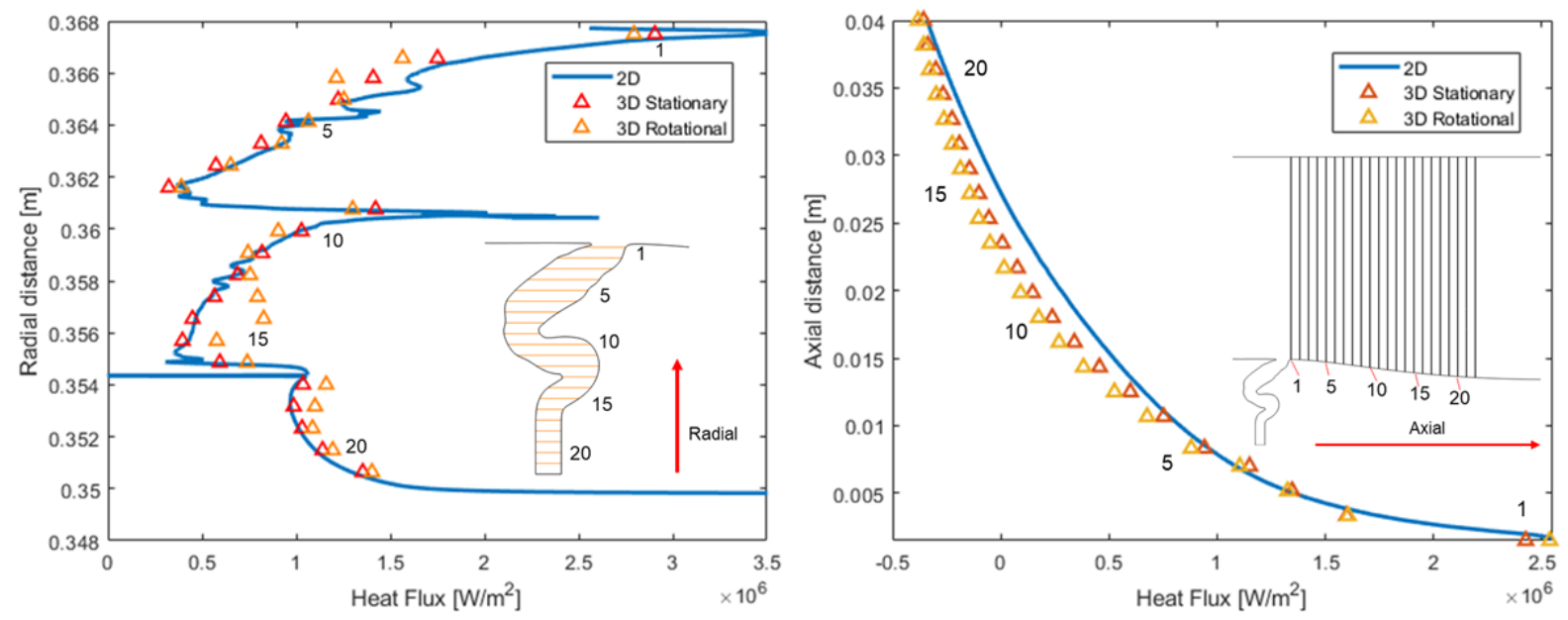

Fig. 22. Left: Heat Flux [W/m2] along the radial location within the cavity. Right: Heat flux on the rotor platform along the axial direction 


\section{CONCLUSION}

This paper demonstrated a methodology for designing the purge geometry based on inspiration from the meandering of rivers. An optimization was performed using URANS to define new designs that provide better cooling of the rotor platform while minimizing purge massflow. The blowing ratio and rotor rear platform temperature appear to be unrelated. Designs that cool the rear platform eject purge flow at lower ejection angles of 20 to 30 degrees. The protective cooling of the rotor platform is clearly a function of the geometric design. Designs that provide better cooling towards the rear platform are also best at cooling the front of the platform. Additionally, designs that allow more massflow also provide higher temperature stability in rear platform. Blockage increases with massflow; however, it can be minimized by allowing the purge to exit at low flow angles.

The design methodology presented in this paper can be used to explore a wide design space and narrow the search for an optimal geometry. Although there are some limitations, the methodology is able predict the trend in heat flux within the cavity and along the platform for both 3D stationary and rotation. The average error in heat flux between $2 \mathrm{D}$ and $3 \mathrm{D}$ is $10 \%$ and $16 \%$ when comparing with $3 \mathrm{D}$ in rotation.

\section{ACKNOWLEDGMENTS}

The authors would like to acknowledge Jorge Saavedra, James Braun, Valeria Andreoli, Danielle Daidone, Udit Vyas, and Zhe Liu for their assistance throughout the preparation of this manuscript.

\section{REFERENCES}

[1] Chilla MM, Hodson HP, Pullan GG, Newman DD. "HighPressure Turbine Rim Seal Design for Increased Efficiency." ASME. Turbo Expo: Power for Land, Sea, and Air Vol. 2B (2016): DOI 10.1115/GT2016-57495.

[2] Pau M, Paniagua G, Delhaye D, de la Loma A, Ginibre P. "Aerothermal Impact of Stator-Rim Purge Flow and RotorPlatform Film Cooling on a Transonic Turbine Stage." ASME. Journal of Turbomachinery Vol. 132 No. 2 (2010): DOI 10.1115/1.3142859.

[3] Paniagua G, Dénos R, Almeida S. "Effect of the Hub Endwall Cavity Flow on the Flow-Field of a Transonic HighPressure Turbine." ASME. Journal of Turbomachinery Vol. 126 No 4 (2004): DOI 10.1115/1.1791644.

[4] Rosic B, Denton JD, Curtis EM, Peterson AT. "The Influence of Shroud and Cavity Geometry on Turbine Performance - An Experimental and Computational Study:

Part II — Exit Cavity Geometry." ASME. Journal of Turbomachinery Vol. 130 No. 4 (2008): DOI 10.1115/1.2777202.
[5] Friedrichs S, Hodson HP, Dawes WN. "Aerodynamic Aspects of Endwall Film-Cooling. ASME. Journal of Turbomachinery Vol. 119 No. 4 (1997): DOI

10.1115/1.2841189.

[6] Barigozzi G, Fontaneto F, Franchini G, Perdichizzi A, Maritano M, Abram R. "Influence of Coolant Flow Rate on Aero-Thermal Performance of a Rotor Blade Cascade With Endwall Film Cooling." ASME. Journal of Turbomachinery Vol. 134 No. 5 (2012): DOI 10.1115/1.4004858.

[7] Zhou DW, Roy RP, Wang CZ, Glahn JA. "Main Gas Ingestion in a Turbine Stage for Three Rim Cavity Configurations." ASME. Journal of Turbomachinery Vol. 133 No. 2 (2010): DOI 10.1115/1.4002423.

[8] Moon M, Lee C, Kim K, Kim J. "Effects of Geometry on Sealing Effectiveness of a Rim Seal." ASME. Turbo Expo: Power for Land, Sea, and Air Vol 5C (2014): DOI 10.1115/GT2014-25368.

[9] Popović I, Hodson HP. "Improving Turbine Stage Efficiency and Sealing Effectiveness Through Modifications of the Rim Seal Geometry." ASME. Turbo Expo: Power for Land, Sea, and Air Vol 4 (2012): DOI 10.1115/GT2012-68026.

[10] Popović I, Hodson HP. “The Effects of a Parametric Variation of the Rim Seal Geometry on the Interaction Between Hub Leakage and Mainstream Flows in High Pressure Turbines." ASME. J. Eng. Gas Turbines Power Vol. 135 No. 11 (2013): DOI 10.1115/1.4024867

[11] McLean C, Camci C, Glezer B. "Mainstream Aerodynamic Effects Due to Wheelspace Coolant Injection in a HighPressure Turbine Stage: Part I-Aerodynamic Measurements in the Stationary Frame." ASME. Journal of Turbomachinery Vol. 123 No. 4 (2001): DOI:10.1115/1.1401026.

[12] Suryanarayanan A, Mhetras SP, Schobeiri MT, Han JC. "Film-Cooling Effectiveness on a Rotating Blade Platform." ASME. Journal of Turbomachinery Vol. 131 No. 1 (2008): DOI 10.1115/1.2752184.

[13] Suryanarayanan A, Ozturk BB, Schobeiri MT, Han JC. Film-Cooling Effectiveness on a Rotating Turbine Platform Using Pressure Sensitive Paint Technique. ASME. Journal of Turbomachinery Vol. 132 No. 4 (2010):

DOI:10.1115/1.3142860.

[14] Cao, C., Chew, J., Millington, P., Hogg, S., "Interaction of Rim Seal and Annulus Flows in an Axial Flow Turbine." ASME. Turbo Expo: Power for Land, Sea, and Air, Turbo Expo Vol 5 (2003): DOI:10.1115/GT2003-38368

[15] Mirzamoghadam, A., Xiao, Z., 2002. "Flow and Heat Transfer in an Industrial Rotor-Stator Rim Sealing Cavity." 
Journal of Engineering for Gas Turbines and Power Vol. 124 No 1 (2002): DOI: 10.1115/1.1400754

[16] Roy, R. et al., "Experiments on Gas Ingestion Through Axial-Flow Turbine Rim Seals." Journal of Engineering for Gas Turbines and Power Vol. 127 No. 3 (2004): DOI: $10.1115 / 1.1850499$

[17] Verstraete, T., "CADO: A Computer Aided Design and Optimization Tool for Turbomachinery Applications," International Conference on Engineering Optimization (2010).

[18] Deb, K., Pratap, A., Agarwal, S., and Meyarivan, T., "A fast and elitist multiobjective genetic algorithm: NSGA-II," IEEE Transactions on Evolutionary Computation Vol. 6 (2002): pp. 182-197.

[19] Juangphanich P, De Maesschalck C, Paniagua G. "From Conceptual 1D Design To Full 3D Optimization of a Highly Loaded Turbine Stage." AIAA. SciTech Forum (2017): DOI 10.2514/6.2017-0110

[20] Clark JP, Grover EA. "Assessing Convergence in Predictions of Periodic-Unsteady Flowfields." ASME. Journal of Turbomachinery Vol. 129 No. 4 (2006): pp.740-749. DOI:10.1115/1.2720504.

[21] Haller, G. "An Objective Definition of a Vortex." Journal of Fluid Mechanics (2005): DOI 10.1017/S0022112004002526
[22] Koláŕ, V. "Vortex identification: New requirements and limitations." International Journal of Heat and Fluid flow (2007): pp.638-652.

[23] J.C.R. Hunt, A.A. Wray, P. Moin. "Eddies, stream, and convergence zones in turbulent flows." Center for Turbulence Research Report CTR-S88 (1988): pp.193-208.

[24] Pinilla V., Solano J.P., Paniagua G., Anthony R., "Adiabatic wall temperature evaluation in a high speed turbine". Journal of Heat Transfer Vol. 134, No. 9 (2012): pp 1-9. DOI: 10.1115/1.4006313.

[25] Solano J.P., Pinilla V., Paniagua G., Lavagnoli S., Yasa T., "Aero-thermal investigation of a multi-splitter axial turbine". International Journal of Heat and Fluid Flow. Vol. 32 No 5 (2011): pp 1036-1046. DOI:

10.1016/j.ijheatfluidflow.2011.05.011

[26] Churchill, S.W., Ozoe, H. "Correlations for forced convection with uniform heating in flow over a plate and in developing and fully developed flow in a tube." Journal of Heat Transfer Vol. 95 (1973) pp. 78-84.

[27] Bhatti, M.S., Shah, R.K. "Turbulent and transition flow convective heat transfer in ducts." In: Kaka, S., Shah, R.K., Aung, W. (Eds.), Handbook of Single-Phase Convective Heat Transfer. Wiley-Interscience, New York. (1987) 